\title{
From terahertz surface waves to spoof surface plasmon polaritons
}

\author{
Yuehong Xu${ }^{1}$, Ying Zhang ${ }^{1}$, Quan $\mathrm{Xu}^{1}$, Xueqian Zhang ${ }^{1}$, Yanfeng $\mathrm{Li}^{1}$, Jianqiang $\mathrm{Gu}^{1}$, Zhen Tian ${ }^{1}$, \\ Chunmei Ouyang ${ }^{1}$, Xixiang Zhang ${ }^{2}$, Weili Zhang ${ }^{1,3}$, Jiaguang Han ${ }^{1}$ \\ ${ }^{1}$ Center for Terahertz Waves and Collegae of Precision Instrument and Optoelectronics Engineering, Tianjin \\ University, 300072 China \\ ${ }^{2}$ Physical Science and Engineering Division, King Abdullah University of Science and Technology, \\ Thuwal 23955-6900, Saudi Arabia. \\ ${ }^{3}$ School of Electrical and Computer Engineering, Oklahoma State University, Stillwater, Oklahoma \\ 74078, USA.
}

\begin{abstract}
Surface plasmon polaritons (SPPs) promise versatile potential applications in many aspects and thus have been a subject of enormous interest. However, in the terahertz regime, due to perfect conductivity of most metals, it is hard to realize a strong confinement of SPPs although a propagation loss could be sufficiently low. Here we introduce the recent work from terahertz surface waves to spoof SPPs based on metasurfaces.
\end{abstract}

\section{INTRODUCTION}

$\mathrm{S}$ urface plasmon polaritons (SPPs), a special form of electromagnetic excitations propagating along a metal/dielectric interface, have been widely exploited in various scientific communities, ranging from physics, chemistry to biology.${ }^{[1]}$ Owing to a confinement of the wave to the surface and an enhancement of the optical field, SPPs are very attractive in developing next-generation, ultra-compact integrated plasmonic circuitry in which light and electric signals can be transferred and processed simultaneously. In an effort to realize subwavelength waveguides and compact integrated circuits, it is vital to excite and control SPPs. This is undoubtedly of great interest not only for the optical frequency, but also for the lower frequencies. However, from optical to the far infrared and terahertz $(\mathrm{THz})$ range, the frequency dependent metal dielectric constant undergoes drastic change. At $\mathrm{THz}$ frequencies, since most metals behave as perfect electrical conductors, the surface does not support bound modes of $\mathrm{THz}$ surface waves. Therefore, for a long time, SPPs have been considered to exist only at optical frequencies. What is the real situation? Yes, at the planar interface of metal/dielectric, terahertz SPPs could not be confined very well and propagate as a half-free-space wave and we call it terahertz surface waves (SWs). In order to realize high-confinement SPPs at $\mathrm{THz}$ frequencies, corrugated metallic structures could be used to support and propagate the SPPs, named spoof SPPs. Here we show the recent results from controllable terahertz surface waves to spoof SPPs based on metasurfaces.

\section{RESULTS}

First, polarization-switchable anomalous launching of SWs and wavefront shaping are theoretically and experimentally demonstrated in a terahertz near-filed metasurface platform. ${ }^{[2]}$ We directly apply the concept of phase discontinuities on SW launching with metasurfaces that consist of metallic aperture resonators. The phase control is realized through interference between a pair of well-arranged apertures on a metal surface. By properly designing the spatial separation and orientation of the paired aperture resonators, nearly arbitrary phase profile is achieved that allows anomalous (directional and focusing/diverging) SW launching and manipulation of the wavefront. This unique approach would also enable promising applications in polarization sensitive terahertz imaging and sensing. The plasmonic mechanism developed in the terahertz regime could also be expanded to the broad spectrum of electromagnetic waves.

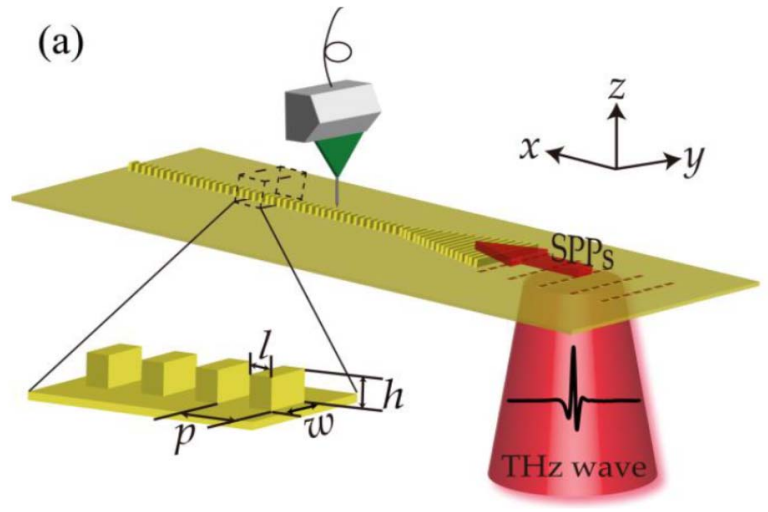

Fig. 1a. Illustration of designed spoof SPPs and experimental measured

Secondly, we theoretically and experimentally demonstrated a $\mathrm{THz}$ spoof SPP waveguide structure consisting of periodic metallic square pillars. ${ }^{[3]}$. Different waveguide components to be investigated included a straight waveguide, an S-bend, a Y-splitter and directional couplers. We identify directly and quantitatively the propgation, bending, splitting and coupling of spoof SPPs at THz frequencies. Figure 1a shows the waveguide structure that we chose was a row of periodic metal pillars arrayed on the metal surface, as well as the measurement methods. Our presented results definitely show that such a structure can be designed arbitrarily to achieve fruitful controlling of spoof SPPs. This kind of plasmonic structure with good functionalities would lead to the development of more robust and complex THz functional devices.

\section{REFERENCES}

[1].W. L. Barnes, A. Dereux, and T. W. Ebbesen, "Surface Plasmon Subwavelength Optics," Nature 424, 824-830, 2003.

[2].X. Zhang, et al., "Anomalous Surface Wave Launching by Handedness Phase Control," Advanced Materials 27, 7123-7129, 2015.

[3].Y. Zhang, et al., "Terahertz spoof surface-plasmon-polariton subwavelength waveguide," Photonics Research 6, 18-23, 2017. 